\title{
Critical behavior and scaling in trapped systems
}

\author{
Massimo Campostrini and Ettore Vicari \\ Dipartimento di Fisica dell'Università di Pisa and I.N.F.N., Largo Bruno Pontecorvo 2, I-56127 Pisa, Italy
}

(Dated: April 2, 2009)

\begin{abstract}
We study the scaling properties of critical particle systems confined by a potential. Using renormalization-group arguments, we show that their critical behavior can be cast in the form of a trap-size scaling, resembling finite-size scaling theory, with a nontrivial trap critical exponent $\theta$, which describes how the correlation length $\xi$ scales with the trap size $l$, i.e., $\xi \sim l^{\theta}$ at $T_{c}$. $\theta$ depends on the universality class of the transition, the power law of the confining potential, and on the way it is coupled to the critical modes. We present numerical results for two-dimensional lattice gas (Ising) models with various types of harmonic traps, which support the trap-size scaling scenario.
\end{abstract}

PACS numbers: 05.70.Jk, 67.85.-d, 64.60.F-.

Recent experimental developments, namely the achievement of Bose-Einstein condensation in dilute atomic vapors of ${ }^{87} \mathrm{Rb}$ and ${ }^{23} \mathrm{Na}$ [1, 2], have attracted great interest in the study of atomic systems in a trapping potential. In the Bose-Einstein condensation scenario, a macroscopic number of trapped bosonic atoms accumulate in a single quantum state and can be described by a condensate wave function, which naturally provides the order parameter of the phase transition. The critical behavior of a trapped Bose gas has been recently investigated experimentally [3], observing an increasing correlation length compatible with the behavior expected at a continuous transition in the three-dimensional XY universality class, see, e.g., Ref. [4] and references therein, which is also the universality class of the superfluid transition in ${ }^{4} \mathrm{He}$, see, e.g., Ref. [5]. However, the inhomogeneity due to the trapping potential strongly affects the phenomenology of phase transitions observed in the absence of a trap. For example, correlation functions of the critical modes are not expected to develop a diverging length scale in a trap. Therefore, a theoretical description of the critical correlations in systems subject to confining potentials, and of how they unfold approaching the transition point, is of great importance for experimental investigations of the critical behavior of systems of trapped interacting particles.

We consider a trapping potential

$$
U(r)=v^{p}|\vec{r}|^{p} \equiv(|\vec{r}| / l)^{p},
$$

where $v$ and $p$ are positive constants and $l=v^{-1}$ is the trap size, coupled to the particle number. Harmonic potentials, i.e., $p=2$, are usually realized in experiments. The effect of the trapping potential is to effectively vary the local value of the chemical potential, so that the particles cannot run away. Let us consider the case in which the system parameters, such as temperature, pressure and chemical potential, are tuned to values corresponding to the critical regime of the unconfined system, where the correlation length diverges as $\xi \sim t^{-\nu}$ and the correlation function behaves as $G(r) \sim 1 / r^{d-2+\eta}$ at $T_{c}\left(t \equiv T / T_{c}-1\right.$ is the reduced temperature and $\nu$ and $\eta$ are the critical exponents of the universality class of the transition). In the presence of a confining potential, the critical behavior of the unconfined homogeneous system could be observed around the middle of the trap only in a window where $\xi$ is much smaller than the trap size but sufficiently large to show the universal scaling behavior. If $\xi$ is large but not much smaller than the trap size, the critical behavior gets somehow distorted by the trap, although it may give rise to universal effects controlled by the universality class of the phase transition of the unconfined system, similarly to finite-size scaling in homogeneous systems of finite size [6, 7]. In the present paper we investigate the critical behavior of trapped systems, putting on a quantitative ground the above qualitative scenario, and in particular the relevant effects of the confining potential. Using renormalization-group (RG) arguments, we show that the critical behavior of the trapped system can be cast in the form of a critical trap-size scaling, resembling standard finite-size scaling theory for homogeneous systems at a continuous transition [6, 7], but characterized by a further nontrivial trap critical exponent.

For the sake of demonstration, as a simple model of trapped particles, one may consider the $d$-dimensional lattice gas model defined by the Hamiltonian

$$
\mathcal{H}_{\text {Lgas }}=-4 J \sum_{\langle i j\rangle} \rho_{i} \rho_{j}-\mu \sum_{i} \rho_{i}+\sum_{i} 2 U\left(r_{i}\right) \rho_{i}
$$

where the first sum runs over the nearest-neighbor sites of the lattice, $\rho_{i}=0,1$ depending if the site is empty or occupied by the particles, $\mu$ is the chemical potential, and $U(r)$ is the potential (11). Far from the origin the potential diverges, thus the expectation value of the particle number tend to vanish, and therefore the particles are trapped. The lattice gas model (2) can be exactly mapped to a standard Ising model, by replacing $s_{i}=1-2 \rho_{i}$, thus $s_{i}= \pm 1$, obtaining

$$
\mathcal{H}=-J \sum_{\langle i j\rangle} s_{i} s_{j}-h \sum_{i} s_{i}-\sum_{i} U\left(r_{i}\right) s_{i}
$$

where $h=2 q J+\mu / 2$ ( $q$ is the lattice coordination number). In the absence of the trap, this model shows a criti- 
cal behavior characterized by a diverging length scale, at the critical point $T=T_{c}$ and $h=h_{c}=0$. Critical correlations do not develop a diverging length scale in the presence of the confining potential, i.e., at fixed $v>0$. We want to study how the critical behavior is distorted by the trap, and how it is recovered in the limit $v \rightarrow 0$.

Our starting point is a scaling Ansatz which extends the scaling law of the RG theory of critical phenomena [8] (see also Refs. [4, 9]), to allow for the confining potential (11). We consider a standard general scenario in which the transition of the unconfined $d$-dimensional system is characterized by two relevant parameters, $t \equiv T / T_{c}-1$ and $h$, as in models (2-3). We write the scaling law of the singular part of the free energy density as

$$
F\left(u_{t}, u_{h}, u_{v}, x\right)=b^{-d} F\left(u_{t} b^{y_{t}}, u_{h} b^{y_{h}}, u_{v} b^{y_{v}}, x / b\right)
$$

where $b$ is any positive number, $x$ is the distance from the middle of the trap, $u_{t, h, v}$ are scaling fields associated with $t, h$, and $v$ respectively. They are analytic functions of the system parameters, behaving as $u_{t} \sim t, u_{h} \sim h$, and $u_{v} \sim v$ when $t, h, v \rightarrow 0 . y_{t, h, v}$ are the corresponding RG dimensions: $y_{t}=1 / \nu$ and $y_{h}=(d+2-\eta) / 2$, while $y_{v}$ must be determined (see below). We are neglecting irrelevant scaling fields, because they do not affect the asymptotic behaviors. Then, fixing $u_{v} b^{y_{v}}=1$ and introducing the trap size $l=v^{-1}$, we obtain

$$
F=l^{-\theta d} \mathcal{F}\left(u_{t} l^{\theta y_{t}}, u_{h} l^{\theta y_{h}}, x l^{-\theta}\right)
$$

where $\theta \equiv 1 / y_{v}$ is the trap exponent. From Eq. (5) one can derive the trap-size scaling of other observables. A generic quantity $S$ is expected to behave asymptotically, when $|t| \rightarrow 0$ and in the large- $l$ limit, as

$$
S=l^{-\theta y_{s}} f_{s}\left(t l^{\theta / \nu}\right)=l^{-\theta y_{s}} \bar{f}_{s}\left(\xi l^{-\theta}\right),
$$

where $y_{s}$ is its RG dimension, $f_{s}$ and $\bar{f}_{s}$ are universal functions (apart from normalizations). $\xi$ is the correlation length, which behaves as $\xi \sim l^{\theta}$ at $T_{c}$, and as $\xi \sim t^{-\nu}$ when $l \rightarrow \infty$. Note that $\bar{f}_{s}(x) \sim x^{-y_{s}}$ for $x \rightarrow 0$, so that $S \sim \xi^{-y_{s}}$ when $l \rightarrow \infty$, which is the scaling behavior in the absence of the trap. These results are quite general, they apply to the lattice gas model (2), as well as to more general interacting gas systems, fluids, etc...

The trap exponent $\theta$ can be computed by analyzing the RG properties of the corresponding perturbation at the critical point. In the case of the model (2), it can be represented by $P_{U}=\int d^{d} x U(x) \phi(x)$, where $\phi(x)$ is the order-parameter field of the $\phi^{4}$ theory which describes the behavior of the critical modes, see, e.g., Ref. [9]. Since the RG dimensions of the potential $U(x)$ and the field $\phi$, respectively $y_{U}=p y_{v}-p$ and $y_{\phi}=\beta / \nu=(d-2+\eta) / 2$, are related by $y_{U}+y_{\phi}=d$, we obtain

$$
\theta=\frac{1}{y_{v}}=\frac{2 p}{d+2-\eta+2 p}
$$

Notice that $\theta \rightarrow 1$ when $p \rightarrow \infty$, which is consistent with the fact that when $p \rightarrow \infty$ the effect of the trapping potential is equivalent to confining a homogeneous system in a box of size $L=l$ with fixed boundary conditions, thus leading to a standard finite size scaling where the RG dimension of the size $L$ is formally minus one [6, 7].

As examples of observables in model (3), we consider the local magnetization and the energy density in the middle of the trap, i.e.,

$$
M_{0} \equiv\left\langle s_{0}\right\rangle, \quad E_{0}=\left\langle s_{0} s_{1}\right\rangle
$$

(where 1 is a nearest neighbor of the center site 0 ), and the correlation function

$$
G_{0}(r) \equiv\left\langle s_{0} s_{r}\right\rangle-\left\langle s_{0}\right\rangle\left\langle s_{r}\right\rangle
$$

In the lattice gas model $M_{0}$ and $G_{0}(r)$ are related to the particle density and its correlations. Their asymptotic trap-size scaling behaviors are given by

$$
\begin{aligned}
& M_{0}=l^{-\theta \beta / \nu} f_{m}\left(t l^{\theta / \nu}\right)=l^{-\theta \beta / \nu} \bar{f}_{m}\left(\xi l^{-\theta}\right), \\
& G_{0}(r)=l^{-\theta \eta} f_{g}\left(t l^{\theta / \nu}, r l^{-\theta}\right)=l^{-\theta \eta} \bar{f}_{g}\left(\xi l^{-\theta}, r / \xi\right), \\
& E_{0}=E_{\mathrm{ns}}(t)+l^{-\theta(d-1 / \nu)} f_{e}\left(t l^{\theta / \nu}\right),
\end{aligned}
$$

where $\beta / \nu=(d-2+\eta) / 2$ and $E_{\mathrm{ns}}(t)$ is a nonsingular function. The scaling behavior at $T_{c}$ can be obtained from the above equations by setting $t=0$,

$$
\begin{aligned}
& M_{0} \sim l^{-\theta \beta / \nu}, \quad E_{0}-E_{c} \sim l^{-\theta(d-1 / \nu)}, \\
& G_{0}(r) \sim l^{-\theta \eta} g\left(r l^{-\theta}\right) .
\end{aligned}
$$

In order to check the trap-size scaling scenario, we consider the square-lattice Ising model (3) with $J=1$ (for which we know the critical temperature and energy, i.e., $T_{c}=2 / \ln (\sqrt{2}+1)$ and $E_{c}=\sqrt{2}$, and the critical exponents, $\nu=1, \eta=1 / 4)$ in a harmonic trap, i.e., the potential (1) with $p=2$. According to Eq. (7), the corresponding trap exponent is $\theta=16 / 31$. Beside two-dimensional (2D) traps, we also consider one-dimensional (1D) traps, where the confining potential acts only along one direction, i.e., $U(r)=v^{p}|x|^{p}$ depends only on $x$, while there is translation invariance along the other $y$ direction. Note that the trap-size scaling formulae, including the value of the trap exponent $\theta$, apply to both 2D and 1D traps.

We performed several Monte Carlo (MC) simulations around the critical point and for various values of the trap size $l$. The lattice size $L$ and harmonic potential of the simulated systems were chosen to have the spin variables effectively frozen at the boundary, making unnecessary the use of larger lattices [10]. The MC results for $M_{0}$ and $E_{0}$ at $T_{c}$ provide accurate checks of the trap-size scaling predicted by Eqs. (13). In Figs. 1 we plot $\bar{M}_{0} \equiv l^{2 / 31} M_{0}$ and $\overline{\Delta E} \equiv l^{\theta}\left(E_{0}-E_{c}\right)$ versus $l^{-1}$, which is the order of the expected leading corrections to the asymptotic scaling [11]. The lines represent fits of all available data, i.e., for $L=2^{n}$ with $n=3, \ldots, 8$, to $\bar{M}_{0}=a+b / l$ (in all cases 


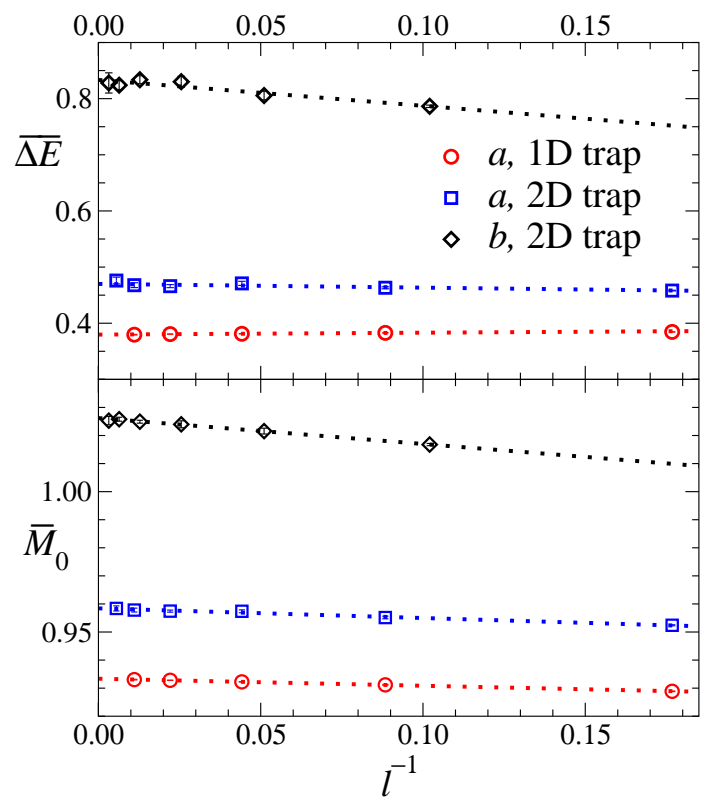

FIG. 1: Trap-size scaling of $M_{0}$ and $E_{0}$ at $T_{c}$ : we plot $\bar{M}_{0} \equiv$ $l^{\theta \eta / 2} M_{0}$ (below) and $\overline{\Delta E} \equiv l^{\theta}\left(E_{0}-E_{c}\right)$ (above) vs $l^{-1}$, for the model (2) with $1 \mathrm{D}$ and $2 \mathrm{D}$ harmonic traps (denoted by " $a$ ") for which $\theta=16 / 31$, and the model (15) with a $2 \mathrm{D}$ harmonic trap (by " $b$ ") for which $\theta=2 / 3$. The dotted lines show linear fits.

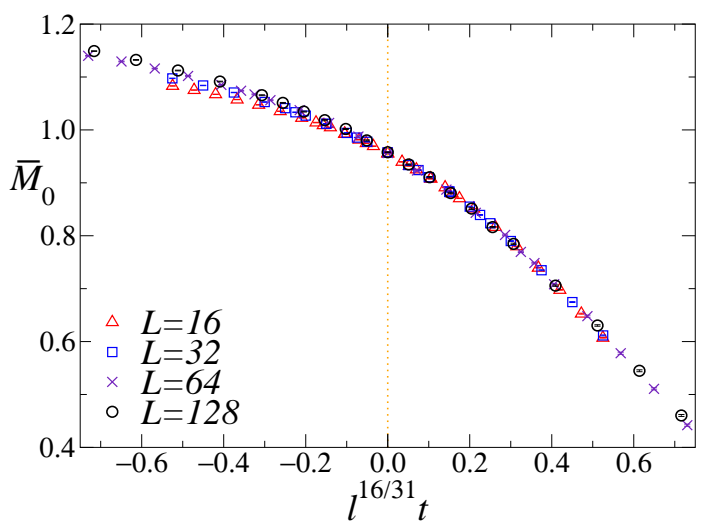

FIG. 2: Trap-size scaling of $M_{0}$ for the model (2) in a 2D trap. $\bar{M}_{0} \equiv l^{2 / 31} M_{0}$ and $L=\sqrt{2} l$.

with $\chi^{2} /$ dof $\lesssim 1$ ). 12] In Fig. 2 we check the scaling of $M_{0}$ around $T_{c}$ in the case of the $2 \mathrm{D}$ trap, by plotting $\bar{M}_{0} \equiv l^{2 / 31} M_{0}$ versus $t l^{16 / 31}$. As predicted by Eq. (10), the data approach a scaling function $f_{m}\left(t l^{16 / 31}\right)$ in the large- $l$ limit, where scaling corrections are suppressed. Fig. 3 shows results for the correlation function (9) in the case of the $2 \mathrm{D}$ trap, at $T_{c}$. They clearly support Eq. (14), indeed the data of $\bar{G}_{0}(r) \equiv l^{4 / 31} G_{0}(r)$ follow the same scaling function $g\left(r l^{-16 / 31}\right)$ for all trap sizes (scaling corrections are very small and not visible in Fig. 3). $G_{0}(r)$ appears to decay rapidly at large distance, with a length scale behaving as $\xi \sim l^{\theta}$.

To further check the trap-size scaling scenario, we also

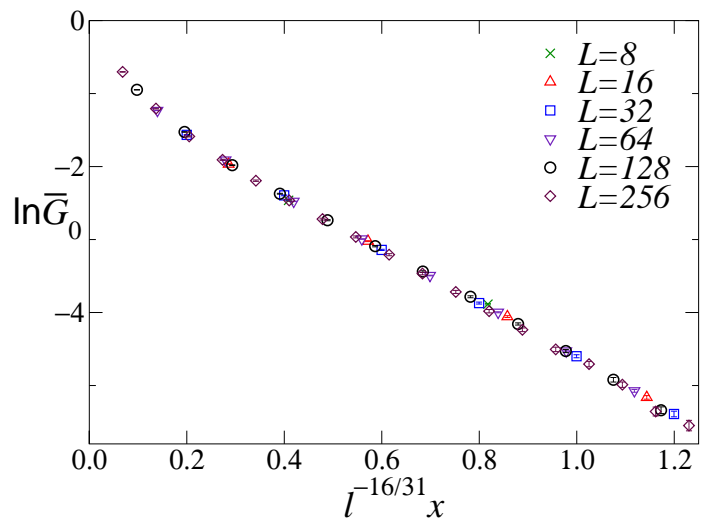

FIG. 3: Scaling at $T_{c}$ of $G_{0}(r)$ for the model (2) in a 2D trap. $\bar{G}_{0}(x) \equiv l^{4 / 31} G_{0}(x)$ and $L=\sqrt{2} l$.

consider another Ising-like model with the confining potential (11) coupled to the energy density, i.e.,

$$
\mathcal{H}_{e}=-J \sum_{\langle i j\rangle}\left[1+U\left(r_{i j}\right)\right] s_{i} s_{j}-h \sum_{i} s_{i}
$$

where the first sum runs over nearest-neighbor sites, and $r_{i j} \equiv\left(r_{i}+r_{j}\right) / 2$. Also in this case the spin variables get frozen at large distances. More precisely, $\lim _{h \rightarrow 0^{+}} \lim _{|r| \rightarrow \infty}\left\langle s_{r}\right\rangle=1$ at any $T$, corresponding to vanishing particle density in the lattice gas model. The effects of the confining potential at the transition can be again described by a trap-size scaling, cf. Eqs. (10,14), but with a different trap exponent $\theta$. Indeed, a RG analysis of the perturbation arising from the potential $U$ in model (15), $Q_{U}=\int d^{d} x U(x) \phi^{2}(x)$, gives [13]

$$
\theta=\frac{p \nu}{1+p \nu}
$$

We again consider a square-lattice model with a harmonic $2 \mathrm{D}$ trap. Since $\nu=1$, Eq. (16) gives $\theta=2 / 3$, which differs from the value $16 / 31$ for the model (3). We performed MC simulations similarly to the previous case. Results for the trap-size scaling of the magnetization $M_{0}$ and the energy $E_{0}$ at the origin, and the correlation function (9) are shown in Figs. 1, 4 and 5. They again fully support the predicted trap-size scaling behaviors. [14]

In conclusion, we have shown that the critical behavior of systems in a confining potential can be described by a universal trap-size scaling (expected to be largely independent of the microscopic details of the model), characterized by a trap exponent $\theta$ which describes how the correlation length scales with the trap size, $\xi \sim l^{\theta}$ at $T_{c}$. The exponent $\theta$ essentially depends on the universality class of the transition, the power law of the confining potential, and on the way it is coupled to the critical modes. These results are very general, and by no means limited to two dimensions.

We finally discuss the critical behavior of a $3 d$ interacting Bose gas trapped by a harmonic potential, 


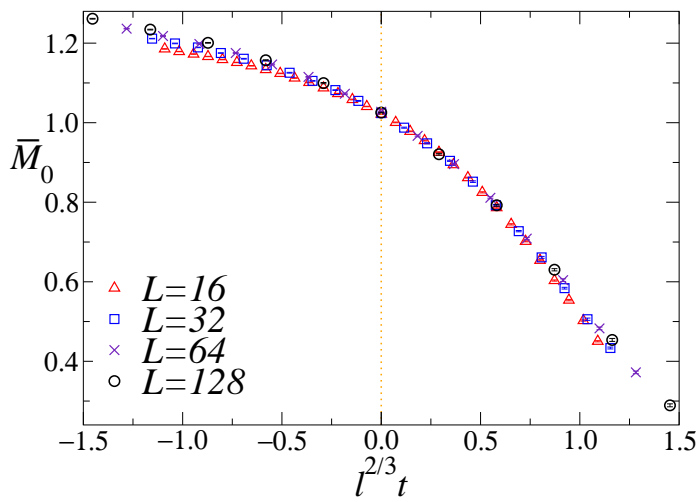

FIG. 4: Trap-size scaling of $M_{0}$ for the model (15) in a 2D trap. $\bar{M}_{0} \equiv l^{1 / 12} M_{0}$ and $L=\sqrt{2 / 3} l$.

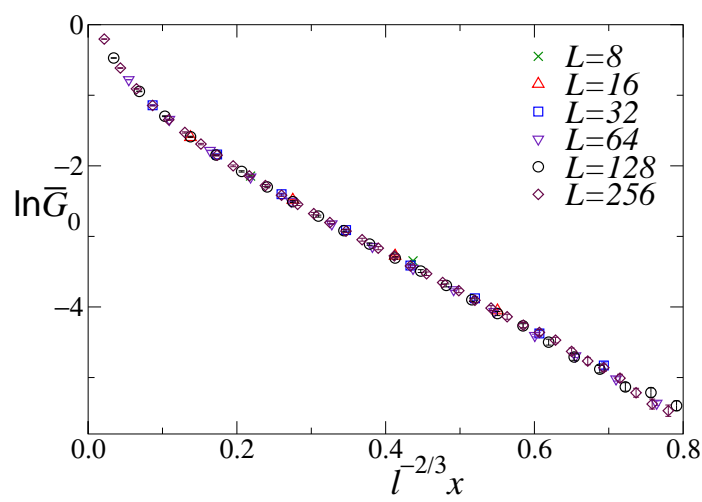

FIG. 5: Scaling at $T_{c}$ of $G_{0}(r)$ for the model (15). $\bar{G}_{0}(x) \equiv$ $l^{1 / 6} G_{0}(x)$ and $L=\sqrt{2 / 3} l$.

which has been recently investigated experimentally [3]. This system is expected to undergo a continuous transition in the $3 d \mathrm{XY}$ universality class, characterized by a complex order parameter $\phi(x)$ with $U(1)$ symmetry. The confining potential $U(x)$ is coupled to the particle density. The corresponding perturbation is [15] $Q_{U}=$ $\int d^{3} x(|x| / l)^{2}|\phi(x)|^{2}$, where $\phi(x)$ is the order-parameter field. The same RG arguments which led us to Eq. (16) give $\theta=2 \nu /(1+2 \nu)$, thus $\theta=0.57327(4)$ using [16] $\nu=0.6717(1)$. This implies that one can neglect the trap effects only when the correlation length satisfies $\xi \ll l^{\theta}=l^{0.573}$. The experimental results of Ref. 3] were likely taken in this region, and led to the estimate $\nu=0.67(13)$, by fitting the data to the standard behavior $\xi \sim t^{-\nu}$. For larger values of $\xi$, when $\xi$ and $l^{\theta}$ become comparable, the trap-size scaling discussed in this paper is expected to provide the correct critical behavior. We believe that experiments probing the trap-scaling regime can be very interesting, analogously to experiments probing finite-size scaling behavior in ${ }^{4} \mathrm{He}$ at the superfluid transition [17]. Moreover, accurate studies of the critical properties of trapped systems, to check universality and determine the critical exponents, require a robust control of the effects of the confining potential. In this respect, one may actually exploit trap-size scaling, using it to infer the critical exponents from the data, analogously to finite-size scaling techniques for the accurate determination of the critical parameters, see, e.g., Ref. [4].

Helpful discussions with E. Arimondo, P. Calabrese and M. Oberthaler are gratefully acknowledged.

[1] E.A. Cornell, C.E. Wieman, Rev. Mod. Phys. 74, 875 (2002).

[2] N. Ketterle, Rev. Mod. Phys. 74, 1131 (2002).

[3] T. Donner, S. Ritter, T. Bourdel, A. Öttl, M. Köhl, T. Esslinger, Science 315, 1556 (2007).

[4] A. Pelissetto, E. Vicari, Phys. Rep. 368, 549 (2002).

[5] J.A. Lipa, D.R. Swanson, J.A. Nissen, T.C.P. Chui, U.E. Israelsson, Phys. Rev. Lett. 76, 944 (1996).

[6] M.E. Fisher, M.N. Barber, Phys. Rev. Lett. 28, 1516 (1972).

[7] M.N. Barber, in C. Domb, J.L. Lebowitz (Eds.), Phase Transitions and Critical Phenomena, Vol. 8, Academic Press, New York, 1983.

[8] K.G. Wilson, in Nobel Lectures in Physics 1981-1990, G. Ekspong (Ed.), World Scientific Publ. Co., Singapore, 1993.

[9] J. Zinn-Justin, Quantum Field Theory and Critical Phenomena (Clarendon Press, Oxford, 1996).

[10] In the case of the $2 \mathrm{D}$ trap we considered lattices with $-L \leq x, y \leq L$, and trap potential $U_{L}(x)=c\left(x^{2}+\right.$ $\left.y^{2}\right) / \overline{L^{2}}$, while at the boundaries $|x|,|y|=L+1$ the spin variable was kept fixed: $s_{i}=1$. In most simulations we fixed $c=2$, i.e., $L=\sqrt{2} l$. In the case of the $1 \mathrm{D}$ trap, we considered $L_{x} \times L_{y}$ lattices with $U_{L}(x)=c x^{2} / L_{x}^{2}$, and $L_{y} / L_{x} \gg 1$ with periodic boundary conditions along $y$.

[11] Leading scaling corrections arise from nonlinear dependence of the scaling field $u_{v}$ on $v$, i.e., its $O\left(v^{2}\right)$ term. Corrections due to irrelevant perturbations are more suppressed, because their leading RG exponent is $\omega=2$ in the two-dimensional Ising model [4].

[12] Unbiased fits of the data of $M_{0}$ to $a l^{-\kappa}(1+b / l)$ (leaving free the exponent $\kappa$ ) give $\kappa=0.0646(1)$ and $\kappa=$ $0.0649(4)$ for the $1 \mathrm{D}$ and $2 \mathrm{D}$ trap respectively, fully supporting the prediction $2 / 31=0.064516 \ldots$ Fits of the data of $E_{0}$ to $E_{0}-E_{c}=a l^{-\kappa}(1+b / l)$ give $\kappa=0.518(1)$ and $\kappa=0.517(5)$ respectively for the $1 \mathrm{D}$ and $2 \mathrm{D}$ trap, in good agreement with the exact value $\theta=16 / 31=0.5161 \ldots$

[13] $\phi^{2}(x)$ represents the energy density operator in the corresponding $\phi^{4}$ theory, i.e. $H=\int d^{d} x\left[\left(\partial_{\mu} \phi\right)^{2}+r \phi^{2}+u \phi^{4}\right]$. We obtain Eq. (16) from the RG relation $y_{U}+y_{\phi^{2}}=d$ and the fact that the RG dimension of $\phi^{2}(x)$ is $y_{\phi^{2}}=d-y_{t}$.

[14] Fits of the data of $M_{0}$ and $E_{0}-E_{c}$ at $T_{c}$ to $a l^{-\kappa}(1+b / l)$ give $\kappa=0.0834(8)$ and $\kappa=0.675(10)$ respectively, in agreement with the corresponding exact values $1 / 12=$ $0.0833 \ldots$ and $2 / 3$ respectively.

[15] K. Damle, T. Senthil, S.N. Majumdar, S. Sachdev, Europhys. Lett. 36, 7 (1996).

[16] M. Campostrini, M. Hasenbusch, A. Pelissetto, E. Vicari, Phys. Rev. B 74, 144506 (2006).

[17] F.M. Gasparini, M.O. Kimball, K.P. Mooney, M. DiazAvilla, Rev. Mod. Phys. 80, 1009 (2008). 